
und Atemnot

_ Eine 76-jährige Frau hatte zunehmende Probleme beim Schlucken von festen Speisen und eine Atemnot entwickelt. Ein Bariumbreischluck zeigte eine Impression des proximalen Ösophagus von dorsal her, in der Höhe des Aortenbogens. Der Befund ließ an eine aberrierende rechte Arteria subclavia denken (Abb. 1A). Mittels kontrastverstärktem CT wurde die Diagnose bestätigt. Es stellte sich eine aneurysmatisch erweiterte rechte Arteria subclavia dar, die als letzter Ast aus dem Aortenbogen atypisch abging. Die anatomische Situation konnte in einem dreidimensionalen CT-Bild klar dargestellt werden (Abb. 1B).

Eine aberrierende rechte Arteria subclavia ist eine relativ häufige kongenitale Anomalie mit einer Prävalenz von bis zu 1,8\%. Etwa ein Drittel der Betroffenen leidet

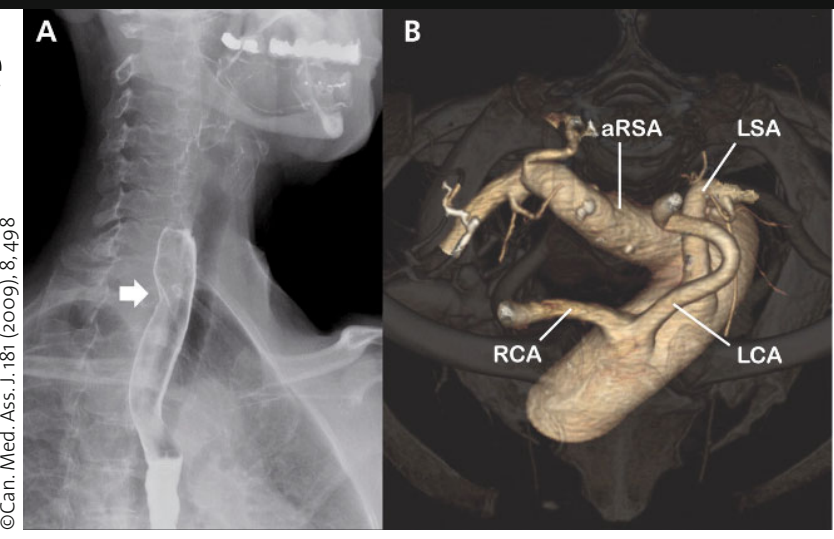

4 Die ÖsophagusImpression (A) deutet auf eine aberrierende rechte Arteria subclavia hin, die im dreidimensionalen CT (B) deutlich sichtbar wird.

unter Symptomen, darunter vor allem einer Dysphagie. Diese Form der Dysphagie wird als Dysphagia lusoria bezeichnet. Dieser Terminus geht auf die erste Beschreibung der Veränderung durch David Bayford im Jahr 1794 zurück, der diesen atypischen Arterienabgang als „Lusus naturae“, d.h. eine Laune der Natur bezeichnete. Es ist nicht ungewöhnlich, dass betroffene Personen die Symptome erst im höheren Lebensalter entwickeln, da sich die aberrierend abgehende Arterie zunehmend aneurysmatisch erweitert und zu einer Einengung des im Alter weniger flexiblen Ösophagus führt. Die bei der Patientin vorhandene Dyspnoe rührt wahrscheinlich von einer Bronchialkompression durch die aberrierende Arterie oder einer Kompression der Arteria pulmonalis her. Bei der Patientin wurde ein rechtsseitiger Bypass zwischen Arteria subclavia und Arteria carotis vorgenommen, worauf die Dysphagie und die Dyspnoe vollständig verschwanden.

H. S. FüEßL =

- J. Dandelooy et al.

Dysphagia lusoria. Can. Med. Ass. J. 181 (2009), 8, 498

\title{
Ginseng gegen die Abgeschlagenheit von Krebspatienten
}

Italienische Forscher haben untersucht, ob sich die chronische Abgeschlagenheit von Krebspatienten mit Ginseng mildern lässt.

— Für die Studie wurden 290 Krebspatienten in vier Gruppen randomisiert. Diese erhielten täglich entweder 0 (Placebo), 0,75, 1,0 oder 2,0 g Panax-Ginseng über acht Wochen. Ihre Abgeschlagenheit wurde mittels des „Brief Fatigue Inventory“ quantifiziert.

Es zeigte sich, dass im Vergleich zur Niedrigdosierung oder zu Placebo die beiden höheren Dosen dieses Symptom effektiver angingen. In den Ver- umgruppen waren etwa doppelt so viele Patienten mit der Behandlung zufrieden als in der Placebogruppe. Ernste Nebenwirkungen wurden nicht berichtet.

\section{Kommentar}

Viele Krebspatienten klagen über eine fast lähmende Abgeschlagenheit, die die Lebensqualität häufig sehr deutlich reduziert. Das Symptom kann sowohl vom Krebs selbst, als auch von der Therapie verursacht werden. Therapeutisch ist es oft nur schwer in den Griff zu bekommen. Zahlreiche ,alternative" Verfahren werden angeboten, aber keines ist mit wirklich überzeugenden Daten belegt.
Die hier vorgelegte Studie ist daher ein Lichtblick. Insbesondere ihre große Fallzahl überzeugt. Ferner scheint sich eine Art Dosis-Wirkungs-Beziehung abzuzeichnen, was natürlich positiv zu bewerten ist. Dennoch, so meine ich, sollten wir zurückhaltend bleiben: Wir benötigen weitere Studien, bevor Ginseng als Mittel der Wahl in die Routine Einzug finden kann.

E. ERNST =

\section{- D. L. Barton et al.}

Pilot study of Panax quinquefolius (American ginseng) to improve cancer-related fatigue: a randomized, double-blind, dose-finding evaluation: NCCTG trial No3CA. Support. Care Cancer. 18 (2010) 179-187 